\section{PTH-009 DOES IMAGING THE OESOPHAGUS, STOMACH AND DUODENUM WITH THE ESO 2 PILLCAM COMPARE FAVOURABLY WITH CONVENTIONAL VIDEO- ENDOSCOPY IN PATIENTS WITH DYSPEPSIA?}

doi:10.1136/gut.2011.239301.410

LMarelli, ${ }^{1,}{ }^{*}$ L Jackson, ${ }^{1}$ F Jaboli, ${ }^{1}$ M Hamilton, ${ }^{1} 0$ Epstein ${ }^{1}$ Centrefor Gastroenterology, Royal Free Hospital, London, UK

Introduction Oesophageal capsule endoscopy (ESO 2) has been developed to image the oesophageal mucosa and compares with endoscopy (OGD). ${ }^{1}$ With a battery life of $30 \mathrm{~min}$, the capsule transmits gastric and even duodenal images beyond its designated function. Trituration causes the capsule to tumble back and forth between fundus and antrum, before passing through the pylorus. With its fore and aft cameras providing a 312 degree field of vision, it is likely that ESO could visualise most, if not all the gastric mucosa. The authors' aim was to compare accuracy of ESO versus OGD for detecting mucosal pathology in patients with uncomplicated dyspepsia and/or heartburn, and patient discomfort.

Methods 50 consecutive outpatients, referred with dyspepsia and/or heartburn ingested an ESO 2 PillCam and underwent OGD $4 \mathrm{~h}$ later. ESO was reported independently by 2 experienced capsule readers and OGD was performed by an expert endoscopist, blinded to ESO results, and recorded on DVD. At the end of the study, a final report was agreed by 2 gastroenterologists viewing ESO and recorded OGD simultaneously, side by side, with biopsy results. This defined benchmark against which to judge the initial ESO and OGD reports. Each patient completed a questionnaire assessing ESO versus OGD discomfort.

Results 50 patients (mean age 56 \pm 15 ; M/F: 17/33) had OGD, 35 without sedation. ESO was ingested by 49 patients (1 unable to swallow the capsule). The duodenum was visualised in 30 of 49 (61\%). The final (benchmark) reports identified major pathology in 15 patients (6 Barretts, 1 pyloric channel ulcer, 5 oesophagitis, 6 erosive gastritis and 4 large hiatus hernia) and in 17 minor pathology (13 superficial gastritis, 10 small hiatus hernia and 4 hyperplastic fundal polyps). 17 patients had normal finding. Compared with the benchmark report, sensitivity and specificity of the initial report for major pathology was $93 \%$ and $100 \%$ for ESO and $87 \%$ and $100 \%$ for OGD. ESO failed to report 1 oesophagitis and OGD failed to detect 1 pyloric ulcer and 1 Barretts oesophagus. Sensitivity and specificity for minor pathology was $76 \%$ and $97 \%$ for ESO and $76 \%$ and $78 \%$ for OGD. ESO had reduced sensitivity for small hiatus hernia and OGD had poor specificity for superficial gastritis. Median patient discomfort (scale 0-10) was 0 for ESO and 4.5 for OGD. Conclusion This pilot study suggests that ESO provides an accurate and well-tolerated method to visualise oesophageal and gastric mucosa, and in $61 \%$, duodenal mucosa. An ESO capsule programmed to broadcast for up to $2 \mathrm{~h}$ could offer a minimally invasive method for imaging the oesophagus, stomach and duodenum. Further studies are necessary to fully evaluate the role of capsule OGD in investigating foregut symptoms.

Competing interests None.

Keywords dyspepsia, oesophageal capsule endoscopy, upper endoscopy.

\section{REFERENCE}

1. Eliakim R, Yassin K, Shlomi l, et al. A novel diagnostic tool for detecting oesophageal pathology: the PillCam oesophageal video capsule. Aliment Pharmacol Ther 2004;20:1083-9. 